Pis'ma v ZhETF

\title{
Mapping deflections of extragalactic Ultra-High Energy Cosmic Rays in magnetohydrodynamic simulations of the Local Universe
}

\author{
Klaus Dolag ${ }^{a}$, Dario Grasso ${ }^{b}$, Volker Springel ${ }^{c}$ and Igor Tkachev ${ }^{d}$ \\ ${ }^{a}$ Dipartimento di Astronomia, Università di Padova, Padua, Italy \\ ${ }^{b}$ Scuola Normale Superiore and I.N.F.N., Pisa, Italy \\ ${ }^{c}$ Max-Planck-Institut für Astrophysik, Garching, Germany \\ ${ }^{d} C E R N$ - Theory Division, Geneve, Switzerland
}

\begin{abstract}
We construct a map of deflections of ultra-high energy cosmic rays by extragalactic magnetic fields using a magneto-hydrodynamical simulation of cosmic structure formation that realistically reproduces the positions of known galaxy clusters in the Local Universe. Large deflection angles occur in cluster regions, which however cover only an insignificant fraction of the sky. More typical deflections of order $\lesssim 1^{\circ}$ are caused by crossings of filaments. For protons with energies $E \geq 4 \times 10^{19} \mathrm{eV}$, deflections do not exceed a few degrees over most of the sky up to a propagation distance of $500 \mathrm{Mpc}$. Given that the field strength of our simulated intergalactic magnetic field forms a plausible upper limit, we conclude that charged particle astronomy is in principle possible.
\end{abstract}

PACS: $98.70 . \mathrm{Sa}$

Introduction. Considerable effort is presently undertaken around the world to build [1, 2] experiments de"voted to determining the composition, the energy spectrum and the arrival directions of Ultra High Energy Cosmic Rays (UHECR). This challenge is in part motivated by the Greisen-Zatsepin-Kuzmin (GZK) puzzle 3] which became particularly acute with Fly-Eye and AGASA data 4, and by the realization that the UHECR flux at $E>10^{19} \mathrm{eV}$ is probably dominated by 'the emission of sources which are quite different from conventional galactic sources. The directional informa'tion may allow the identification of UHECR sources, provided primary particles are not deflected too much by galactic and intergalactic magnetic fields (IGMFs).

Several arguments suggest that UHECR are electrically charged nuclei, most probably they are protons. The possibility of neutral particles is not ruled out, but needs not be discussed here since such rays point back to the sources anyway. It is possible that a fraction of UHECR is comprised of iron nuclei, see e.g. Ref. [5]. However, according to an analysis of inclined events recorded by the Haverah Park shower detector [6], above $10^{19} \mathrm{eV}$ less than $30 \%$ of the primary cosmic rays can be iron nuclei at the $95 \%$ confidence level. In what follows we normalize our results to the case of protons. The case of other nuclei can be recovered by multiplication with their charge, $\mathrm{Z}$.

Galactic magnetic fields (MF) with $B_{\text {gal }} \sim 1 \mu \mathrm{G}$ are not expected to produce significant deflections at extremely high energies, $E \gtrsim 10^{20} \mathrm{eV}$ in the case of protons. Even at lower energies $E \sim 4 \times 10^{19} \mathrm{eV}$, strategies have been proposed which allow source identification without detailed knowledge of the galactic MF [7.

The very attractive perspective to do astronomy with proton primaries might however be spoiled by the presence of strong IGMFs. So far, evidences of the presence of IGMFs have been found only within, or very close to, rich clusters of galaxies. The most relevant observations are those based on Faraday rotation measurements (RM) of the polarized radio emission of sources located within or behind clusters, and on the synchrotron emission of relativistic electrons in the intracluster MF. The results of both methods imply the presence of MFs with strength at the $\mu \mathrm{G}$ level extending up to $1 \mathrm{Mpc}$ from cluster centers. The coherence length of the field is inferred to lie in the range $10-100 \mathrm{kpc}$ (see recent review [8] and references therein). Such fields do certainly induce large deflections of UHECR protons that cross clusters of galaxies. However, galaxy clusters fill only a tiny fraction of the volume of the universe, so that we may expect them to produce large deflections at best over a small portion of the sky [9, 10]. Outside clusters, only upper limits on the IGMF strength are available. They are at the level of $1-10 \mathrm{nG}$ for fields extending over cosmological distances with coherence lengths in the range 50 to $1 \mathrm{Mpc}$, respectively [1]. These bounds do not hold for MFs in clustered regions, like filaments connecting galaxy clusters where the field might be as large as $10^{-7} \mathrm{G}$. In principle, either a weak all pervading smooth field, or stronger fields localized in a complex web of filaments, may produce sizable deflections of UHECR over a large portion of the sky. It is 
hence evident that a better knowledge of the large-scale magnetic structure of the universe is called for.

In this letter, we approach this problem by means of numerical simulations of cosmic structure formation, where we combine the collisionless dynamics of the dark matter component with the magnetohydrodynamics (MHD) of the magnetized gas. Our basic hypothesis is that the MFs observed in rich clusters of galaxies are the outcome of a MHD amplification process powered by the hierarchical formation of clusters. This assumption is supported by the results of previous simulations which, under the same hypothesis that we adopt here, succeeded to reproduce the general features of RM in several observed clusters [12]. The tiny seed field required to initiate the amplification process may be either of primordial origin [13] or the result of a battery associated with the initial stages of structure formation 14.

Simulations of the magnetic structure of the universe and of the UHECR propagation within it have been previously attempted by several authors [14, 15, 16. A novel achievement of our work is that we have performed constrained simulations, which reproduce the observed large scale structures in the nearby universe, leaving essentially no ambiguity for the choice of observer position. This is quite relevant in the present context, since it has been shown [16] that the angular distribution, as well as the energy spectrum, of UHECRs reaching an observer located in a weakly magnetized region may differ considerably from that seen by a strongly magnetized one. Furthermore, by tracing UHECR trajectories in the simulated magnetic structures we are able to construct maps of expected UHECR deflections as a function of distance that, for the first time, account for the actual large-scale structure as seen from the Galaxy.

MHD simulations of the Local Universe. We use initial conditions that were constructed from the IRAS 1.2-Jy galaxy survey by first smoothing the observed galaxy density field on a scale of $7 \mathrm{Mpc}$, evolving it linearly back in time, and then using it as a Gaussian constraint for an otherwise random realization of the $\Lambda \mathrm{CDM}$ cosmology. In [19] it was shown that these constrained initial conditions, when evolved to the present time, reproduce the observed density and velocity field of the Local Universe. In addition, they allow a direct identification of prominent clusters (Virgo, Coma, Centaurus, Hydra, Perseus, A3627, and Pavo) with counterparts formed in the simulation, which are found at the right places, and with approximately the correct observed masses. We extended the initial conditions of [19] by adding gas, together with an initial MF. The volume filled by high resolution particles within our simulation is a sphere of radius $\sim 115 \mathrm{Mpc}$ centered on the Milky Way. This region comfortably includes the entire Local Super Cluster (LSC) and is modelled with a maximum spatial resolution of $10 \mathrm{kpc}$. The simulation uses 51 million gas particles of mass $6.9 \times 10^{8} \mathrm{M}_{\odot}, 51$ million highresolution dark matter particles, and an additional 7 million boundary particles in the distant low-resolution region.

We evolved the initial conditions with the newest version of the GADGET-code 17, adding the Magnetic Smoothed Particle Hydrodynamics (MSPH) technique [12 to follow MF evolution. Previous work [12] showed that magnetic seed fields in the range of $(1-5) \times 10^{-9} \mathrm{G}$ at redshift $z_{*} \simeq 20$ will be amplified due to the structure formation process and reproduce RM in clusters of galaxies. This corresponds to $B\left(z_{*}\right)\left(1+z_{*}\right)^{-2} \simeq 0.2-1 \times 10^{-11} \mathrm{G}$ at the present time in the unclustered intergalactic medium (IGM). It was also demonstrated that the MF amplification process completely erases any memory of the initial field configuration in high density regions like galaxy clusters. Therefore, we can safely set the coherence length $l_{c}\left(z_{\text {in }}\right)$ of the initial seed field to be infinite in our simulation. Although this assumption is probably unrealistic, it does not lead to underestimation of the UHECR deflections. Concerning the initial strength of the MF, we used the highest value which still allowed previous MSPH simulations to successfully reproduce RM in clusters, i.e. the results presented here give safe upper bounds on UHECR deflections.

Clusters are generally connected by magnetized filamentary structures of gas and dark matter, where highdensity filaments often harbor small clusters or groups. We find that shock fronts and shear flows are ubiquitous in these filaments, giving rise to substantial MHD amplification in these structures, boosting the MF intensity far above the expectation of adiabatic compression alone, as pointed out in previous work [12. We also identified low density filaments where the MF is roughly aligned along their axis, with a strength of $\sim 10^{-4} \mu \mathrm{G}$. This is consistent with a purely adiabatic amplification of the seed MF due to the compression of field lines. We find no significant MF in the neighborhood of the Milky Way's position, which is contrained to lie within a sphere of $7 \mathrm{Mpc}$ around the origin. We find a group of four halos aligned within the super galactic plane in this region, corresponding to the Local Group. Due to the lack of small-scale constraints it is not certain which of the four galaxies should be best associated with the Milky Way, but this does not affect our results. Because it is a small galaxy group, the MFs associated with the 


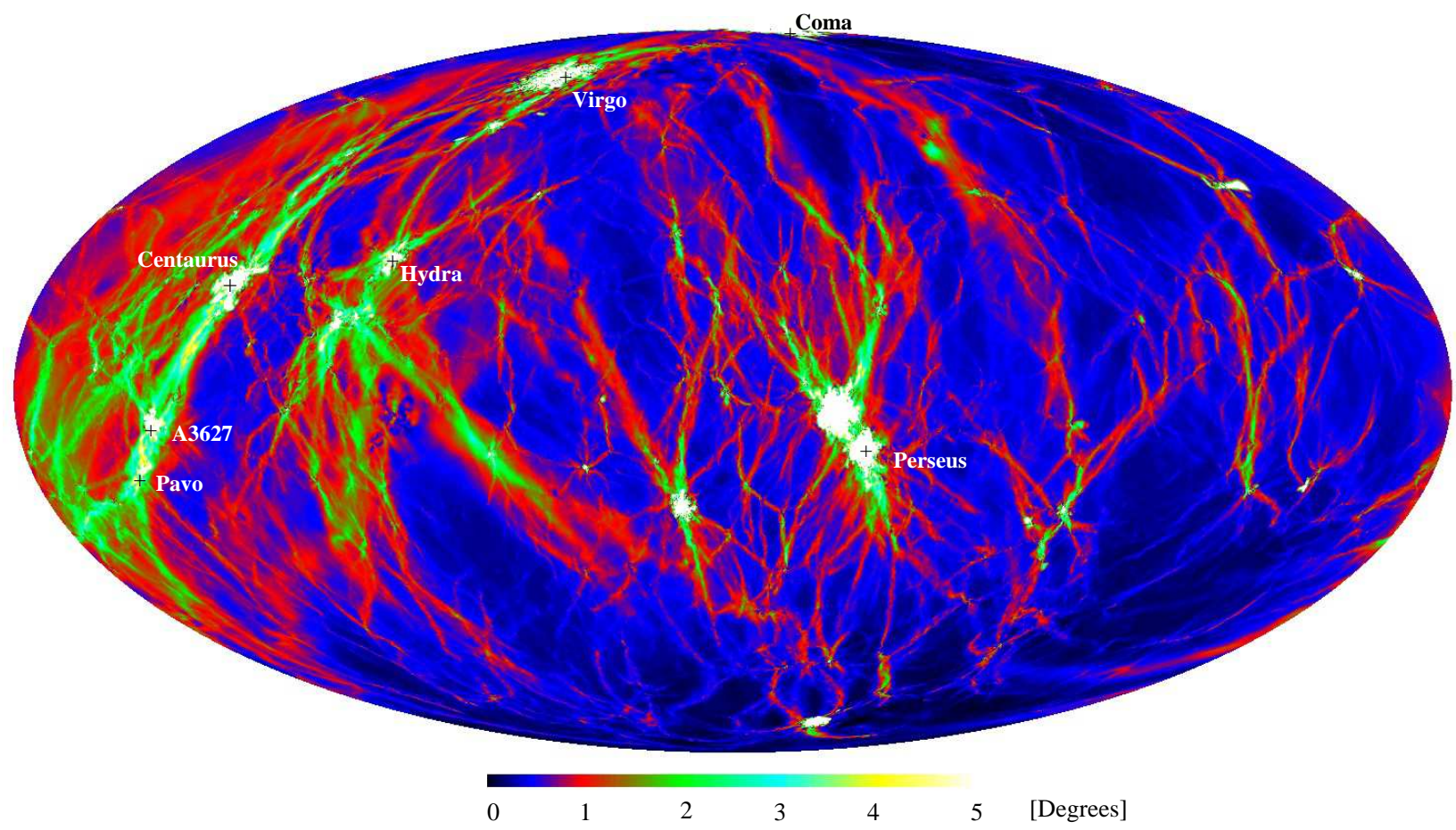

FIG. 1. Full sky map (area preserving projection) of deflection angles for UHECRs with energy $4 \times 10^{19} \mathrm{eV}$ using a linear color scale. All structure within a radius of $107 \mathrm{Mpc}$ around the position of the Galaxy was used. The coordinate system is galactic, with the galactic anti-center in the middle of the map. Positions of identified clusters are marked using the locations of the corresponding halos in the simulation. Note that deflections internal to the Milky Way have not been included.

group are sufficiently weak to not lead to significant deflections, despite covering a large fraction of the sky.

Deflections of charged UHECR. Having obtained a 3D model of MFs in the Local Universe, we can construct an associated map of deflections of charged particles under the action of the Lorentz force. We here consider only protons with energy $E=4 \times 10^{19} \mathrm{eV}$. This is the threshold value for the process of photo-pion production in collisions with Cosmic Microwave Background $(\mathrm{CMB})$ photons $\left(p+\gamma_{C M B} \rightarrow p(n)+\pi^{0(+)}\right)$. The energy loss length is large, $l_{E} \sim 1000 \mathrm{Mpc}$ (for a recent review see e.g. [18]), and initially higher proton energies quickly degrade into this range. Neglecting energy losses and taking $E=4 \times 10^{19} \mathrm{eV}$ to be the energy at detection, we obtain upper bounds for the deflections of protons with higher energy since the deflection angle decreases linearly increasing the energy.

We do not follow particle trajectories directly; instead we compute accumulated deflections along rectilinear paths. This is a reasonable simplification since we are not interested in actual source positions, but rather in finding directions with small deflections. In Fig. 11, we show a deflections map obtained by tracing an isotropic distribution of protons from a maximal distance of $d_{\max }=107 \mathrm{Mpc}$ to the observer. Recall that Fig. 11 represents a map of deflections, not a distribution of arrival directions. The former is independent of the assumed distribution of UHECR sources.

The pattern of clusters and filaments is clearly visible in Fig. 1 Large deflections are produced only when protons cross the central regions of galaxy clusters, and most of these strongest deflections are found along a strip which can be approximately identified with the Great Attractor. The observed positions of Virgo, Coma, Hydra and Centaurus lie in this region. Their locations quite precisely coincide with regions where the deflections exceed $4^{\circ}$. Perseus and other minor clusters produce large deflections in other well delineated regions of the sky. Outside clusters, which occupy only a small fraction of the sky, deflections of $1^{\circ}-2^{\circ}$ occur along an intricate network of filaments, covering a larger area. The regions with $\delta \ll 1^{\circ}$ correspond to voids where the MF strength is even smaller than $10^{-11} \mathrm{G}$.

In order to investigate the relative importance of deflectors at different distance, we also produced deflection maps that only included deflectors up to some maximum distance. We observe no significant deflections produced 
at distances smaller than $7 \mathrm{Mpc}$. Massive clusters at large distances $(\sim 100 \mathrm{Mpc})$ produce large deflections but cover only a negligible fraction of the sky, so that the bulk of the deflections is produced by passages through filaments.

In Fig. 2] we plot the fraction of the sky, $A\left(\delta_{\mathrm{th}}\right)$, over which deflections larger than $\delta_{\text {th }}$ are found, for different propagation distances. We see that deflections larger than $1^{\circ}$ are to be expected over less than $20 \%$ of the sky up to the distance $d=107 \mathrm{Mpc}$. For large distances $d$, we find that $A\left(\delta_{\mathrm{th}}, d\right)$ approaches a self-similar behavior, viz. $A\left(\delta_{\mathrm{th}}, d\right)=A_{0}\left(\delta_{\mathrm{th}} \times\left(d_{0} / d\right)^{\alpha}\right)$. Numerically, we observe $\alpha=0.8$ for $70<d / \mathrm{Mpc}<110$. Selfsimilarity is consistent with the assumption that the density of deflectors (filaments) reaches a constant value at large distances. Since MFs are uncorrelated in different filaments, multiple filament crossings should produce a "random walk" in the deflection angle, resulting in $\alpha=0.5$. The value of $\alpha=0.8$ we observe may hence indicate that the regime of multiple filament crossings is not yet reached over the distances probed by our simulation. We include an extrapolation of $A\left(\delta_{\mathrm{th}}, d\right)$ up to a distance of $500 \mathrm{Mpc}$ in Fig. 2] shown for two values of $\alpha$, the observed one of $\alpha=0.8$, and the expected one for large propagation distances, $\alpha=0.5$. We expect that these two curves bracket the range of true deflections at $E \sim 4 \times 10^{19} \mathrm{eV}$.

We comment finally on the potential effect of the unclustered component of the IGMF, i.e. the field in voids and low density regions outside of clusters and filaments, coherence length of which, $l_{c}$, is unconstrained by our simulations. If $l_{c} \ll d$ the proton trajectory makes a random walk through the magnetic domains, and the overall deflection is given by

$$
\delta \simeq 0.2^{\circ}\left(\frac{B_{0}}{E} \frac{4 \times 10^{19} \mathrm{eV}}{10^{-11} \mathrm{G}}\right)\left(\frac{d}{1 \mathrm{Gpc}}\right)^{\frac{1}{2}}\left(\frac{l_{c}}{1 \mathrm{Mpc}}\right)^{\frac{1}{2}} .
$$

Hence, observable deflections are not produced by the unclustered component of the IGM if $l_{c}$ is smaller than a few tens of Mpc. Note that such small coherence lengths are expected from most of the proposed generation mechanisms of seed IGMFs [13. The few mechanisms predicting larger $l_{c}$ generally give rise to MF which are too weak to produce observable deflections of UHECR. Furthermore, an unclustered IGMF does not exist at all if the seed field is generated by a battery powered by structure formation [14.

Conclusions. We presented the first map of UHECR deflections in the Local Universe that is based on a simulation that realistically reproduces the known largescale structure around the Galaxy, while simultaneously

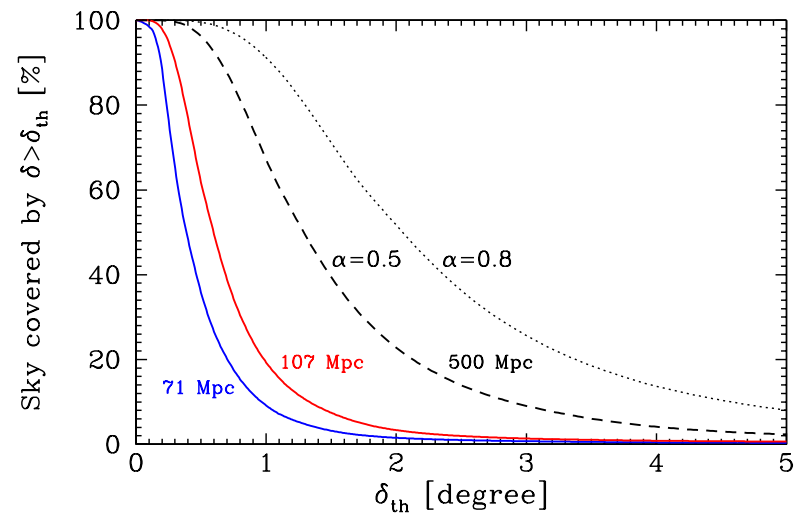

FIG. 2. Cumulative fraction of the sky with deflection angle larger than $\delta_{\mathrm{th}}$, for several values of propagation distance (solid lines). We also include an extrapolation to $500 \mathrm{Mpc}$, assuming self similarity with $\alpha=0.5$ (dashed line) or $\alpha=0.8$ (dotted line). The assumed UHECR energy for all lines is $4.0 \times 10^{19} \mathrm{eV}$.

following the MHD amplification of MFs during cosmic structure formation. The positions and masses of the most prominent clusters are reproduced well in our simulation. This is an important advantage of our technique. Since local structures subtend large angles on the sky, it is important to be able to reliably identify "bad" regions of expected large deflections, a task that can be accomplished using our map, thereby providing important guidance for UHECR source identification. Provided our basic hypothesis about the origin of IGMF is correct, our results should be understood as upper bounds for the expected deflection angles, because we have used the largest seed field still compatible with the RM in clusters, and secondly, we neglected UHECR energy losses on the path to the detector. The actual observation of stronger deflections would imply that the evolution of the IGMF is not merely passive, possibly indicating a pollution of the IGM by physical process such as galactic winds.

We have also extrapolated the distribution of deflection angles to very large source distances in a statistical manner. Out to $500 \mathrm{Mpc}$ and at $E \geq 4 \times 10^{19} \mathrm{eV}$, typical deflections are smaller than the angular resolution of current ground array UHECR detectors over more than half of the sky (but may exceed angular resolution of stereo fluorescent detectors). This result is consistent with an observed small-scale clustering of UHECR arrival directions [20] ${ }^{1)}$ and with evidence for a BL Lacs - UHECR correlation [24] in the energy range $E \sim 4 \times 10^{19} \mathrm{eV}$ being due to protons [25]. On the

\footnotetext{
1) There is no clustering in the current HiRes data 21 22, which became avaliable after submission of our paper. However, with the current statistics there is no contradiction yet [22 23].
} 
other hand, our results do not support models which invoke strong MFs in the local universe to solve the GZK anomaly as well as models which explain smallscale clustering by magnetic lensing.

We conclude that charged particle astronomy should in principle be possible regardless of the way the GZK problem will be resolved.

\section{ACKNOWLEDGMENTS}

We thanks V. Berezinsky, P. Blasi, D. Semikoz, P. Tinyakov, M. Vietri for reading the manuscript and providing useful comments. The simulations were carried out on the IBM-SP4 machine at the 'Rechenzentrum der Max-Planck-Gesellschaft', with CPU time assigned to the 'Max-Planck-Institut für Astrophysik'. Fig. 1 has been produced using HEALPix [26. K. Dolag acknowledges support by a Marie Curie Fellowship of the European Community program 'Human Potential' under contract number MCFI-2001-01227.

1. http://www.auger.org

2. http://www.euso-mission.org

3. K. Greisen, Phys. Rev. Lett. 16, 748 (1966); G.T. Zatsepin and V.A. Kuzmin, Pisma Zh. Eksp. Teor. Fiz. 4, 144 (1966).

4. D. J. Bird et al., Astrophys. J. 441, 144 (1995); M. Takeda et al., Phys. Rev. Lett. 81, 1163 (1998) .

5. A. A. Mikhailov, Pisma Zh. Eksp. Teor. Fiz. 79, 175 (2004).

6. M. Ave, J. A. Hinton, R. A. Vazquez, A. A. Watson and E. Zas, Phys. Rev. Lett. 85, 2244 (2000).

7. P. Tinyakov and I. Tkachev, Proceedings of the 28th International Cosmic Rays Conference, astro-ph/0305363

8. C.L. Carilli and G.B. Taylor, Ann. Rev. Astron. \& Astrophys., 40, 319 (2002).

9. V. Berezinsky, A. Z. Gazizov and S. I. Grigorieva, arXiv:astro-ph/0210095

10. P. Blasi and D. De Marco, arXiv:astro-ph/0307067

11. P. Blasi, S. Burles and A. V. Olinto, Astrophys. J. 514, L79 (1999).

12. K. Dolag, M. Bartelmann and H. Lesch, Astron. \& Astrophys. 387, 383 (2002).

13. D. Grasso and H. R. Rubinstein, Phys. Rept. 348, 163 (2001).

14. R. M. Kulsrud, R. Cen, J. P. Ostriker and D. Ryu, Astrophys. J. 480, 481 (1997).

15. D. Ryu, H. Kang and P. L. Biermann, Astron. \& Astrophys. 335, 19 (1998).

16. G. Sigl, F. Miniati and T. A. Ensslin, Phys. Rev. D 68, 043002 (2003), and arXiv:astro-ph/0309695
17. V. Springel, N. Yoshida, and S.D.M. White, New Astronomy, 6, 79 (2001).

18. L. Anchordoqui, T. Paul, S. Reucroft and J. Swain, Int. J. Mod. Phys. A 18, 2229 (2003).

19. H. Mathis et al., MNRAS, 333, 739 (2002).

20. N. Hayashida et al., Phys. Rev. Lett. 77, 1000 (1996); M. Takeda et al., Astrophys. J. 522, (1999) 225; P. G. Tinyakov and I. I. Tkachev, JETP Lett. 74, 1 (2001) [Pisma Zh. Eksp. Teor. Fiz. 74, 3 (2001)].

21. R. U. Abbasi et al. [The High Resolution Fly's Eye Collaboration (HIRES)], arXiv:astro-ph/0404137

22. R. U. Abbasi et al. [The High Resolution Fly's Eye Collaboration (HiRes)], arXiv:astro-ph/0404366

23. H. Yoshiguchi, S. Nagataki and K. Sato, arXiv:astro-ph/0404411

24. P. G. Tinyakov and I. I. Tkachev, JETP Lett. 74, 445 (2001) [Pisma Zh. Eksp. Teor. Fiz. 74, 499 (2001)].

25. P. G. Tinyakov and I. I. Tkachev, Astropart. Phys. 18, 165 (2002).

26. K.M. Gorski, E. Hivon, B.D. Wandelt, Proceedings of the MPA/ESO Cosmology Conference "Evolution of Large-Scale Structure", eds. A.J. Banday, R.S. Sheth and L. Da Costa, PrintPartners Ipskamp, NL, pp. 37-42, 1999. See also: http://www.eso.org/science/healpix/ 